\title{
Assessment of Quality of Life and Severity of Itching Pre and Post Doxepin Therapy in Dialysis Patients with Pruritus
}

\author{
Kousalya Prabahar ${ }^{1 *}$, Danda Anusha ${ }^{2}$, Soundararajan Periasamy ${ }^{3}$, Boddu Sri Venkata Rishitha ${ }^{2}$, \\ Anand Ramanathan ${ }^{2}$, Dasari Anudeep ${ }^{2}$ \\ ${ }^{1}$ Department of Pharmacy Practice, Faculty of Pharmacy, University of Tabuk, Tabuk, Kingdom of Saudi Arabia. \\ ${ }^{2}$ Department of Pharmacy Practice, Faculty of Pharmacy, Sri Ramachandra University, Chennai, Tamil Nadu, India. \\ ${ }^{3}$ Department of Nephrology, Sri Ramachandra University, Chennai, Tamil Nadu, India.
}

\section{ARTICLE INFO}

Article history:

Received on: 01/12/2016

Accepted on: 23/05/2017

Available online: 30/11/2017

Key words:

Itch; pruritus; doxepin.

\begin{abstract}
Objective: Chronic kidney disease (CKD)-associated pruritus is a significant clinical symptom affecting more than $50 \%$ of patients on hemodialysis. The main aim of this study was to assess the effects of doxepin treatment on the quality of life of pruritic patients.

Material and methods: This prospective-interventional study was carried out for a period of 6 months in dialysis unit of Department of Nephrology in a tertiary care teaching hospital, Chennai. Both inpatients and outpatients of both sexes $\geq 18$ years on hemodialysis or peritoneal dialysis for more than 3 months with pruritus were included for the study. A total number of 39 dialysis patients with pruritus were recruited for the study. Patients were evaluated at the beginning of the study and at the end of 2 weeks of each study period. The severity, duration, degree, direction, disability and distribution of itch were evaluated using 5D-pruritus scale. The quality of life of patients was assessed using DLQI questionnaire (Dermatology Life Quality Index).

Results: There was an improvement in quality of life of patients with pruritus after doxepin therapy.

Conclusion: Doxepin is a better therapy for itching and improving the quality of life in dialysis patients. Clinicians should enquire about skin irritation and itching during consultations since patients may be reluctant to admit to these symptoms due to embarrassment.
\end{abstract}

\section{INTRODUCTION}

Pruritus (skin irritation or itching) is common in patients with Chronic Kidney Disease (CKD) stages 4 and 5. It is associated with disrupted sleep, reduced quality of life, depression and increased mortality (Rayner et al., 2012). Pruritus is a common and bothersome symptom among patients with endstage-renal-disease (ESRD) (Nolan, 2005).Chronic kidney disease (CKD)-associated pruritus is a significant clinical symptom affecting more than $50 \%$ of patients on hemodialysis (Mettang and Weisshaar, 2010). People with end-stage kidney disease have a higher incidence of skin itching than the general population, while dialysis also raises this high incidence.

Dr. Kousalya Prabahar, Assistant Professor, Department of Pharmacy Practice, Faculty of Pharmacy, University of Tabuk, Tabuk, Kingdom of Saudi Arabia. E-mail: happykousi @ gmail.com
Therefore, patients' ESRD, dialysis treatment and phosphorus metabolic disorder all can contribute to their skin problems (Ostlere et al., 1994). In rare cases, this skin problem is due to allergies. If patients notice itching occurs at the beginning of dialysis treatments, they may have an allergy to the blood tubing, dialyzer or other elements associated with dialysis (Francos et al., 1991).If suffering from severe skin itching that happens all the time, the high buildup of wastes may be the root cause. In this situation, kidney function improvement is the most effective treatments to solve both your skin problem and many other symptoms. The key to achieve this purpose is to increase damaged kidney cells' self-curative ability and nourish them. As for the latter two causes, correct medicines, diet and some changes in dialysis treatment can help ease itching effectively (Ostlere et al., 1994; Stockenhuber et al., 1987). Scratching may lead to impetigo (skin infection), prurigo (papules) and chronic, lichenified dermatitis/eczema. 
Because of the ignorance about its pathogenesis it is not surprising that treatment of uremic pruritus is quite unsatisfactory. Treatments currently used for uremic pruritus such as antihistamines, steroids, emollients, and phototherapy (UVB) have not been investigated rigorously, and no drugs have been approved for this indication by the U.S. Food and Drug Administration (Ponticelli and Bencini, 1995). Doxepin, as a H1-receptor blocker, is an effective and safe choice not only in idiopathic pruritus, but also in uremic pruritus resistant to conventional treatments. Doxepin can help improve pruritus resistant to antihistamines in end-stage renal disease patients who undergo hemodialysis (PorReza-Gholi et al., 2007). A low dose of doxepin is safe while effective and its main adverse effect, drowsiness, is temporary and can be easily tolerated by the patients.

Antihistamines are usually ineffective in patients with uremic pruritus (Greene et al., 1985). Hence we found it logical trying an agent with histaminic activity in haemodialysis patients.

Hence the main objectives of the study were to assess the effects of doxepin treatment on the quality of life of pruritic patients and the improvement in severity, distribution and frequency of pruritus related sleep disturbance after doxepin treatment.

\section{MATERIAL AND METHODS}

This prospective-interventional study was carried out for a period of 6 months in dialysis unit of Department of Nephrology in a tertiary care teaching hospital, Chennai, after getting approval from the Institutional Ethics Committee (Ref. No. CSP/13/OCT/31/176). Thirty-nine patients were enrolled in the study. Both inpatients and outpatients of both sexes equal to or above 18 years on hemodialysis or peritoneal dialysis for more than 3 months with antihistamine resistant pruritus were included for the study. Patients with a history of dermatologic disease antedating renal failure and those with skin disease other than cutaneous findings of uremia and with systemic diseases like malignancy, cholestatic liver disease were excluded from the study. Informed consent was obtained from each patient before enrolling in the study. Data on patient demographics (age, sex, body weight, body surface area), stage of chronic kidney disease, drugs prescribed, co-morbidities, surgical and dialysis details were all entered in a patient data collection form. Patients were prescribed with doxepin $10 \mathrm{mg} /$ day at night for a period of 2 weeks. They were evaluated at the beginning of the study - before doxepin therapy and at the end of 2 weeks - after doxepin therapy. Each evaluation included a limited physical examination and assessment of severity of pruritus. Pruritus was auto-assessed by the patients. Each patient was directly asked to fill the questionnaires. The score was assessed by the investigator for all the patients and at each time of study.

The severity, duration, degree, direction, disability and distribution of itch were evaluated using 5D- pruritus scale (Appendix 1). The 5D-pruritus scale comprised of 5 domains that are used to assess the level of itching. The quality of life of patients was assessed using DLQI questionnaire (Dermatology Life Quality Index) (Appendix 2). Both are validated questionnaires. The Dermatology Life Quality Index questionnaire is designed for use in adults, i.e. patients over the age of 16. It is self-explanatory and can be simply handed to the patient who is asked to fill it in without the need for detailed explanation. It is usually completed in one or two minutes. If the patients were illiterate and were not able to understand the questionnaire we assisted them by explaining every domain of the questionnaire either or gave them the questionnaire in their vernacular language.

\section{Statistical methods}

The collected data was statistically analyzed using SPSS 16.0 version software. The results were represented as frequency, percentage, mean and standard deviation wherever applicable. Internal consistency for both the questionnaires was measured using Cronbach's alpha. In addition, Pearson correlation was used to compare the level of significance between post and prior obtained domain scores for both the questionnaires. Comparison of questionnaires before and after treatment was done using Wilcoxon signed rank test.

\section{RESULTS}

A total number of 39 dialysis patients with pruritus who were equal to or above the age of 18years and of either sex from the department of nephrology were recruited for the study. The mean age of the patients was $53.36 \pm 12.811$ (Range 18 to 72 years). Majority of the patients $(n=11,28 \%)$ fall in the age group of 50-59 years followed by 60-69 years $(n=10,25 \%)$.In the study population of $39,12(31 \%)$ were female and $27(69 \%)$ were male. Among them, 6(15\%) were in the stage-3chronic kidney disease and the remaining $33(85 \%)$ were in the stage-5 chronic kidney disease. The major co-morbidity among the study population was hypertension (HTN) - 30(77\%) and diabetes mellitus (DM) $21(54 \%)$. Among the study population of 39,30 patients $(77 \%)$ had itching for a period of less than 6 months (Table 1).

Table 1: Patient characteristics.

\begin{tabular}{|c|c|c|}
\hline & & Frequency (number) \\
\hline \multirow[t]{6}{*}{ Age (years) } & $<30$ & $8 \%(3)$ \\
\hline & $30-39$ & $8 \%(3)$ \\
\hline & $40-49$ & $23 \%(9)$ \\
\hline & $50-59$ & $28 \%(11)$ \\
\hline & $60-69$ & $25 \%(10)$ \\
\hline & $70+$ & $8 \%(3)$ \\
\hline \multirow[t]{2}{*}{ Gender } & Male & $69 \%(27)$ \\
\hline & Female & $31 \%(12)$ \\
\hline \multirow[t]{2}{*}{ CKD stage } & 3 & $15 \%(6)$ \\
\hline & 5 & $85 \%(33)$ \\
\hline \multirow[t]{8}{*}{ Co-morbidities } & Renal anaemia & $10 \%(4)$ \\
\hline & Hypertension & $77 \%(30)$ \\
\hline & Diabetes Mellitus & $54 \%(21)$ \\
\hline & Glomerulonephritis & $44 \%(1)$ \\
\hline & Rheumatoid Heart Disease & $3 \%(1)$ \\
\hline & Seizure & $3 \%(1)$ \\
\hline & Coronary Heart Disease & $3 \%(1)$ \\
\hline & Urosepsis & $3 \%(1)$ \\
\hline \multirow[t]{3}{*}{ Duration of itch } & $<6$ months & $77 \%(30)$ \\
\hline & 6 months -1 year & $20 \%(8)$ \\
\hline & $>1$ year & $3 \%(1)$ \\
\hline
\end{tabular}


Each domain in the 5D-Pruritus scale was assessed before and after doxepin therapy (Table 2). A statistically significant difference was found in each domain of 5D-Pruritus scale after doxepin therapy. This shows that the duration of itch was diminished, the intensity of itch was reduced, the itching became better, the impact of itch on sleep, social activities, housework and work was lessened and the distribution of itch on the body was greatly decreased after doxepin therapy.

Table 2: Evaluation of 5D - Pruritus scale before and after doxepin therapy.

\begin{tabular}{|c|c|c|c|c|c|c|}
\hline \multirow[b]{2}{*}{ 苞 } & \multicolumn{2}{|c|}{ Q1 Pre } & \multirow[b]{2}{*}{ 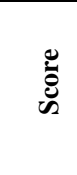 } & \multicolumn{2}{|c|}{ Q1 Post } & \multirow[b]{2}{*}{$=\frac{\mathscr{J}}{\pi}$} \\
\hline & $\frac{\dot{\Xi}}{\bar{\Xi}}$ & 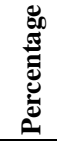 & & $\frac{\dot{\Xi}}{\bar{\Xi}}$ & 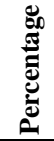 & \\
\hline 1 & 2 & 5 & 1 & 22 & 56 & \\
\hline 2 & 16 & 41 & 2 & 15 & 38 & \\
\hline 3 & 20 & 51 & 3 & 1 & 3 & $0.001^{*}$ \\
\hline 4 & 0 & 0 & 4 & 1 & 3 & \\
\hline \multirow[t]{2}{*}{5} & 1 & 3 & 5 & 0 & 0 & \\
\hline & \multicolumn{2}{|c|}{ Q2 Pre } & & \multicolumn{2}{|c|}{ Q2 Post } & \\
\hline 1 & 0 & 0 & 1 & 5 & 13 & \\
\hline 2 & 13 & 33 & 2 & 27 & 69 & \\
\hline 3 & 18 & 46 & 3 & 6 & 15 & $0.001^{*}$ \\
\hline 4 & 8 & 21 & 4 & 1 & 3 & \\
\hline \multirow[t]{2}{*}{5} & 0 & 0 & 5 & 0 & 0 & \\
\hline & \multicolumn{2}{|c|}{ Q3 Pre } & & \multicolumn{2}{|c|}{ Q3 Post } & \\
\hline 1 & 0 & 0 & 1 & 5 & 13 & \\
\hline 2 & 0 & 0 & 2 & 25 & 64 & \\
\hline 3 & 15 & 38 & 3 & 7 & 18 & $0.001^{*}$ \\
\hline 4 & 23 & 59 & 4 & 2 & 5 & \\
\hline \multirow[t]{2}{*}{5} & 1 & 3 & 5 & 0 & 0 & \\
\hline & \multicolumn{2}{|c|}{ Q4a Pre } & & \multicolumn{2}{|c|}{ Q4a Post } & \\
\hline 1 & 1 & 3 & 1 & 1 & 3 & \\
\hline 2 & 5 & 13 & 2 & 26 & 66 & \\
\hline 3 & 12 & 31 & 3 & 8 & 21 & $0.001^{*}$ \\
\hline 4 & 12 & 31 & 4 & 2 & 5 & \\
\hline \multirow[t]{2}{*}{5} & 9 & 23 & 5 & 2 & 5 & \\
\hline & \multicolumn{2}{|c|}{ Q4b Pre } & & \multicolumn{2}{|c|}{ Q4b Post } & \\
\hline N/A & 1 & 3 & N/A & 1 & 3 & \\
\hline 1 & 12 & 31 & 1 & 25 & 64 & \\
\hline 2 & 12 & 31 & 2 & 10 & 25 & $0001^{*}$ \\
\hline 3 & 10 & 25 & 3 & 3 & 8 & 0.001 \\
\hline 4 & 4 & 10 & 4 & 0 & 0 & \\
\hline \multirow[t]{2}{*}{5} & 0 & 0 & 5 & 0 & 0 & \\
\hline & \multicolumn{2}{|c|}{ Q4c Pre } & & \multicolumn{2}{|c|}{ Q4c Post } & \\
\hline N/A & 1 & 3 & N/A & 1 & 3 & \\
\hline 1 & 10 & 26 & 1 & 25 & 64 & \\
\hline 2 & 13 & 33 & 2 & 9 & 23 & $0001^{*}$ \\
\hline 3 & 9 & 23 & 3 & 4 & 10 & 0.001 \\
\hline 4 & 6 & 15 & 4 & 0 & 0 & \\
\hline 5 & 0 & 0 & 5 & 0 & 0 & \\
\hline & & & & & & \\
\hline N/A & 28 & 72 & N/A & 28 & 72 & \\
\hline 1 & 4 & 10 & 1 & 5 & 13 & \\
\hline 2 & 4 & 10 & 2 & 5 & 13 & 0461 \\
\hline 3 & 2 & 5 & 3 & 1 & 3 & 0.461 \\
\hline 4 & 1 & 3 & 4 & 0 & 0 & \\
\hline 5 & 0 & 0 & 5 & 0 & 0 & \\
\hline & & & & & & \\
\hline 1 & 7 & 18 & 1 & 25 & 64 & \\
\hline 2 & 32 & 82 & 2 & 14 & 36 & \\
\hline 3 & 0 & 0 & 3 & 0 & 0 & $0.001^{*}$ \\
\hline 4 & 0 & 0 & 4 & 0 & 0 & \\
\hline 5 & 0 & 0 & 5 & 0 & 0 & \\
\hline
\end{tabular}

The DLQI questionnaire was assessed before and after doxepin therapy (Figure 1). A statistically significant difference was found in the scores after doxepin therapy $(\mathrm{p}<0.05)$. This shows that the quality of life was very much improved after doxepin therapy.

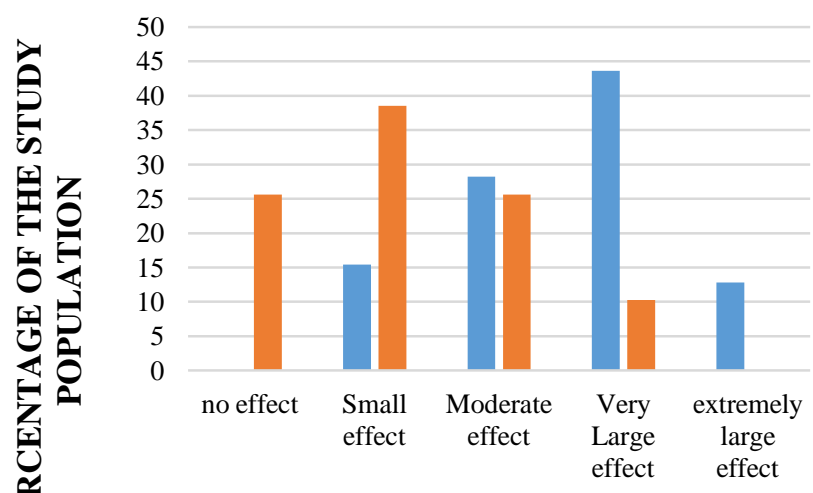

Effect on patient's qualty of life based on DLQI Scores.

ఐBEFORE DRUG ADM. $\square$ AFTER DRUG ADM.

Fig. 1: Assessment of DLQI questionnaire before and after doxepin therapy.

Each domain in the 5D-Pruritus scale and each question in the DLQI questionnaire was compared before and after doxepin therapy using Wilcoxon signed rank test. Overall, there was a statistically significant difference found in almost all the questions (Table 3).Based on Wilcoxon signed rank test, positive ranks and the sum of negative ranks equals the sum of positive ranks, analysis was done to compare the significant difference in each component of both questionnaires before and after the treatment.From the table, it was inferred that there were significant positive outcomes from doxepin therapy. The questions DLQI6 and Q4D were answered "not relevant" to this particular study population and so there was no significant difference.

Table 3: Comparison of the questions before and after doxepin therapy using Wilcoxon signed rank test.

\begin{tabular}{|c|c|c|}
\hline Questionnaires & $\mathbf{Z}$ & Asymp. Sig. (2-tailed) \\
\hline Q1 Post - Q1 Pre & $-5.340^{\mathrm{b}}$ & $0.001^{*}$ \\
\hline Q2 Post - Q2 Pre & $-4.767^{\mathrm{b}}$ & $0.001^{*}$ \\
\hline Q3 Post - Q3 Pre & $-5.370^{\mathrm{b}}$ & $0.001^{*}$ \\
\hline Q4a Post - Q4a Pre & $-4.927^{\mathrm{b}}$ & $0.001^{*}$ \\
\hline Q4b Post - Q4b Pre & $-4.064^{\mathrm{b}}$ & $0.001^{*}$ \\
\hline Q4c Post - Q4c Pre & $-4.247^{\mathrm{b}}$ & $0.001^{*}$ \\
\hline Q4d Post - Q4d Pre & $-0.736^{\mathrm{b}}$ & 0.461 \\
\hline Q5 Post - Q5 Pre & $-5.125^{\mathrm{b}}$ & $0.001^{*}$ \\
\hline Total 5D Post - Total 5D Pre & $-5.311^{b}$ & $0.001^{*}$ \\
\hline DLQI Post1 - DLQI Pre1 & $-4.617^{\mathrm{b}}$ & $0.001^{*}$ \\
\hline DLQI Post2 - DLQI Pre2 & $-4.513^{\mathrm{b}}$ & $0.001^{*}$ \\
\hline DLQI Post3 - DLQI Pre3 & $-4.939^{\mathrm{b}}$ & $0.001^{*}$ \\
\hline DLQI Post4 - DLQI Pre4 & $-4.450^{\mathrm{b}}$ & $0.001^{*}$ \\
\hline DLQI Post5 - DLQI Pre5 & $-4.500^{\mathrm{b}}$ & $0.001^{*}$ \\
\hline DLQI Post6 - DLQI Pre6 & $0.000^{\mathrm{c}}$ & 1.000 \\
\hline DLQI Post7 - DLQI Pre7 & $-4.684^{\mathrm{b}}$ & $0.001^{*}$ \\
\hline DLQI Post8 - DLQI Pre8 & $-4.082^{\mathrm{b}}$ & $0.001^{*}$ \\
\hline DLQI Post9 - DLQI Pre9 & $-3.945^{\mathrm{b}}$ & $0.001^{*}$ \\
\hline DLQI Post10 - DLQI Pre10 & $-3.464^{\mathrm{b}}$ & $0.001^{*}$ \\
\hline Total DLQI Post - DLQI Pre & $-5.445^{b}$ & $0.001^{*}$ \\
\hline
\end{tabular}
"p $<0.05$ is statistically significant, b. Based on positive ranks. c. The sum of negative ranks equals the sum of positive ranks. 


\section{DISCUSSION}

Chronic Kidney Disease with Pruritus is the common health problem especially among elderly who undergo dialysis. In our study, a high incidence of Pruritus was observed among the mean age of $53.36 \pm 12.81$. It was similar to the study conducted by Khan TN et al., 2013, in which the mean age was $52 \pm 17.5$ years which showed no difference among the Arab population when compared to our population. In our study, the majority of the patients were men $(69 \%)$ and in a study carried out by Khan et al., 2013 also, the majority of sample involved were men (65\%), which indicates that the incidence of pruritus is greater in men when compared to women. In our study, hypertension $(n=30,77 \%)$ and diabetes mellitus $(\mathrm{n}=21,54 \%)$ were the common comorbid conditions, which was very much similar to the study conducted by Khan et al., 2013. Greene et al., 1985 compared the effect of doxepin and diphenhydramine in systemic form for the treatment of idiopathic urticaria. They used doxepin $10 \mathrm{mg}$ thrice daily and recorded the subjective outcomes, whereas we used doxepin once daily at night and recorded the outcomes using questionnaires. In the present study, we found a significant $(\mathrm{p}<0.05)$ improvement in the quality of life and decrease in severity of pruritus in patients receiving haemodialysis, whereas in a study conducted by PourReza-Gholi et al., 2007, there was a significant effect of doxepin for the treatment of pruritus in ESRD patients receiving haemodialysis, which proves that doxepin can be used in the treatment of treatment-resistant pruritus. In a study conducted by Khan et al., 2013, the reliability of the five domain 5D pruritus scale indicated first rate reliability and was found to be a reliable measure for assessment of pruritus in patients with uremic pruritus and so in our study the 5D pruritus scale was administered to assess the severity of itching. In our study population of 39 $(100 \%)$, pruritus was found mild in $33 \%$, moderate in $46 \%$ and severe in $21 \%$, while in a study conducted by Akhyani et al., 2005, pruritus was found to be mild in $51 \%$, moderate in $11 \%$ and severe in $37 \%$ of patients.

In a study conducted by Welter et al., 2008, DLQI Quality of life assessment revealed that there was no effect of pruritus in $20.3 \%$, small effect in $37.4 \%$, moderate effect in $13 \%$, very large effect in $18.8 \%$ and extremely large effect in $10.1 \%$ of patients on the quality of life. Our study revealed small effect of pruritus in $6(15 \%)$, moderate effect in $11(28 \%)$, very large effect in $17(44 \%)$ and an extremely large effect in $5(13 \%)$ patients.

In a study conducted by Szepietowski et al., 2002, among the demographic variables, only age was found to significantly interfere in a negative manner in the DLQI, i.e. with more severe scores for patients who were younger $(r=-0.20, p=0.0003$; the mean age was $64.21 \pm 0.72$ ). The gender, ESRD causal disease, type of Minimal Renal Disease (MRD) and duration of MRD had no significant impact on DLQI, while in our study the mean age was $52 \pm 17.5$ years, the male population showed greater prevalence than the female study population, also the prevalence in CKD-V $(85 \%)$ patients was greater than patients in CKD-III stage (15\%).
Haemodialysis patients with moderate or severe itch are significantly more likely to feel drained or depressed and have $17 \%$ higher mortality risk, statistically associated with quality of sleep. In our study, the disability caused to the patients due to sleep disturbance was severe (delays falling asleep and occasionally wakes me up at night) in $23 \%$ before the treatment but $5 \%$ of study population reported severe sleep disability after the treatment, which proves that doxepin has relatively good effect in reducing disability in pruritic patients due to insomnia. In a study conducted by Susel et al., 2014, the mean score of DLQI was $3.6 \pm 3.4$ points $(4.4 \pm 4.4$ points in women and $3.2 \pm 2.8$ points in men). There was no significant difference of DLQI score between female and male patients with Uremic Pruritus. In our study the mean DLQI score before the treatment was $2.59 \pm 0.910$ and it was $1.23 \pm 0.902$ after the treatment which demonstrates a significant improvement in the quality of life in pruritic patients after treatment with doxepin.

In a study conducted by Mathur et al., 2010, 67\% of patients were taking medications for pruritus. Those with greater Uremic Pruritus severity were somewhat more likely to take medications $(56,74$, and $70 \%$ in categories $\mathrm{A}, \mathrm{B}$, and $\mathrm{C}$ respectively). All type $\mathrm{C}$ patients (representing $25 \%$ of the study population) reported that the medications "did not help at all" or only "helped a little," compared with two thirds of type A patients. Consistent with reports of medication ineffectiveness, pruritus intensity was high and associated symptoms were frequent despite medication use among most patients. Among the type $\mathrm{C}$ patients who were not taking pruritus medications, 100\% cited "nothing works" as the reason. In our study $23(59 \%)$ patients reported that their itching was unchanged despite medications before the administration of doxepin, later reported that their itching was much better but still present after the treatment.

In our study, the mean score for disability in social activity due to pruritus was $2.10 \pm 1.046$, which was similar to the study conducted by Khan et al., 2013.

\section{CONCLUSION}

Doxepin as a $\mathrm{H} 1$ receptor blocker orally administered at night at a dose of $10 \mathrm{mg}$, for 2 weeks in haemodialysis patients was proven to improve the quality of life and decrease duration, degree, direction, disability and distribution of pruritus.

The clinicians should enquire about skin irritation and itching during consultations since patients may be reluctant to admit to these symptoms due to embarrassment.

\section{Financial support and sponsorship: None.}

Conflict of Interests: There are no conflicts of interest.

\section{REFERENCES}

Akhyani M, Ganji MR, Samadi N, Khamesan B, Daneshpazhooh M. Pruritus in hemodialysis patients. BMC Dermatol, 2005; 5:7. 
Francos GC, Kauh YC, Gittlen SD, Schulman ES, Besarab A, Goyal S, et al. Elevated plasma histamine in chronic uremia. Effects of ketotifen on pruritus. Int J Dermatol, 1991; 30(12):884-9.

Greene SL, Reed CE, Schroeter AL. Double-blind crossover study comparing doxepin with diphenhydramine for the treatment of chronic urticaria. J Am Acad Dermatol, 1985; 12(4):669-75.

Khan TM, Al-Haider I, Sulaiman SAS, Hassali MA. Linguistic validation of the 5D itching scale to Arabic in patients with end-stage kidney disease. J Ren Care, 2013; 39(4):222-7.

Mathur VS, Lindberg J, Germain M, Block G, Tumlin J, Smith $\mathrm{M}$, et al. A longitudinal study of uremic pruritus in hemodialysis patients. Clin J Am Soc Nephrol, 2010; 5(8):1410-9.

Mettang T, Weisshaar E. Pruritus: Control of itch in patients undergoing dialysis. Skin Therapy Lett, 2010; 15(2):1-5.

Nolan CR. Strategies for improving long-term survival in patients with ESRD. J Am Soc Nephrol, 2005; 16 (Suppl 2):S120-7.

Ostlere LS, Taylor C, Baillod R, Wright S. Relationship between pruritus, transepidermal water loss, and biochemical markers of renal itch in haemodialysis patients. Nephrol Dial Transplant, 1994; 9(9):1302-4.

Ponticelli C, Bencini PL. Pruritus in dialysis patients: a neglected problem. Nephrol Dial Transplant, 1995; 10(12):2174-6.

Pour-Reza-Gholi F, Nasrollahi A, Firouzan A, Esfahani EN, Farrokhi F. Low-dose doxepin for treatment of pruritus in patients on hemodialysis. Iran J Kidney Dis, 2007; 1(1):34-7.

Rayner H, Baharani J, Smith S, Suresh V, Dasgupta I. Uraemic pruritus: Relief of itching by gabapentin and pregabalin. Nephron Clin Pract, 2012; 122(3-4):75-9.
Stockenhuber F, Sunder-Plassmann GS, Balcke P. Increased plasma histamine levels in chronic renal failure. N Engl J Med, 1987; 317(6):386.

Susel J, Batycka-Baran A, Reich A, Szepietowski JC. Uraemic pruritus markedly affects the quality of life and depressive symptoms in haemodialysis patients with end-stage renal disease. Acta Derm Venereol, 2014; 94(3):276-81.

Szepietowski JC, Morita A, Tsuji T. Ultraviolet B induces mast cell apoptosis: a hypothetical mechanism of ultraviolet B treatment for uraemic pruritus. Med Hypotheses, 2002; 58(2):167-70.

Welter EQ, Bonfa R, Petry V, Moreira LL, Weber MB. Relationship between pruritus and quality of life in patients on hemodialysis. An Bras Dermatol, 2008; 83:137-40.

\section{How to cite this article:}

Prabahar K, Danda A, Periasamy S, Boddu R, Ramanathan A, Dasari A. Assessment of Quality Of Life and Severity of Itching Pre and Post Doxepin Therapy in Dialysis Patients with Pruritus. J App Pharm Sci, 2017; 7 (11): 119-125. 


\section{5-D Pruritus Scale}

1. Duration: During the last 2 weeks, how many hours a day have you been itching?



2. Degree: Please rate the intensity of your itching over the past 2 weeks

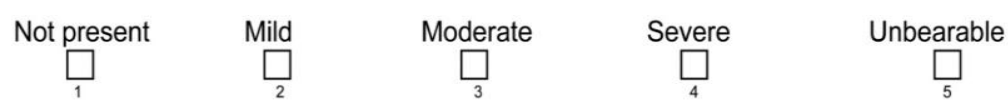

3. Direction: Over the past 2 weeks has your itching gotten better or worse compared to the previous month?

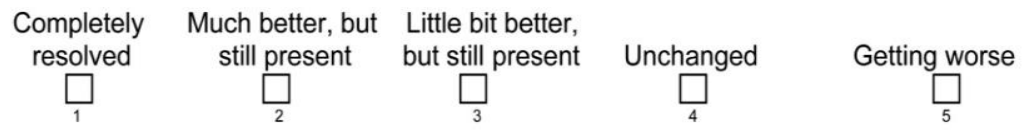

4. Disability: Rate the impact of your itching on the following activities over the last 2 weeks

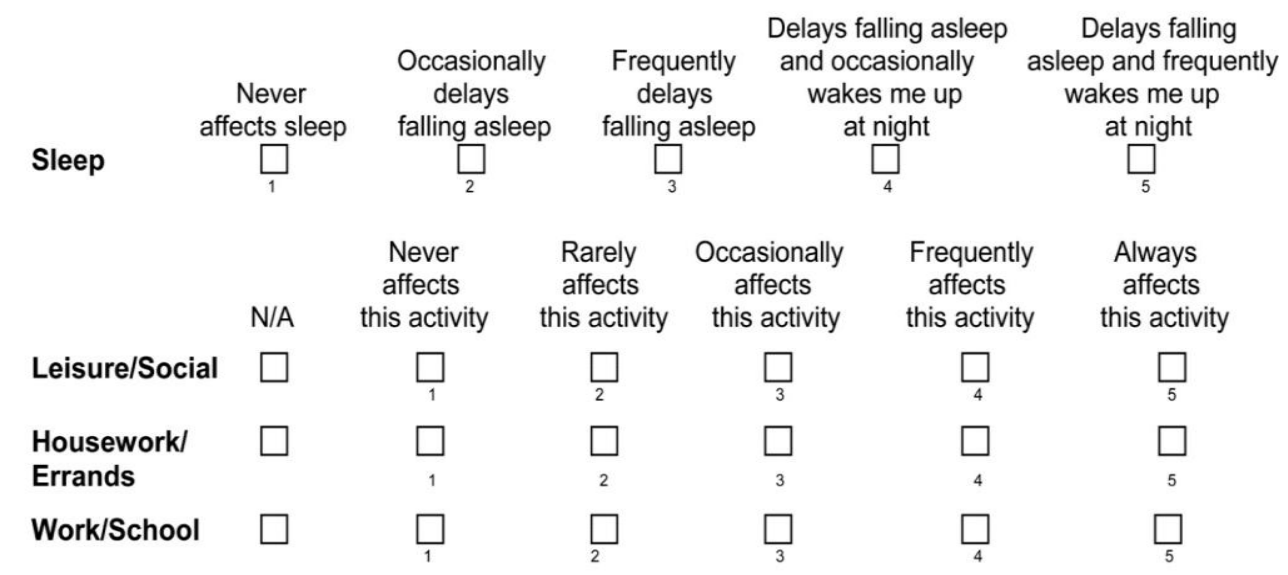

5. Distribution: Mark whether itching has been present in the following parts of your body over the last 2 weeks. If a body part is not listed, choose the one that is closest anatomically.

\begin{tabular}{|c|c|c|}
\hline Head/Scalp & & Soles \\
\hline Face & L & Palms \\
\hline Chest & \begin{tabular}{|l} 
\\
\end{tabular} & Tops of Hands/Fingers \\
\hline Abdomen & L & Forearms \\
\hline Back & 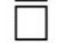 & Upper Arms \\
\hline Buttocks & 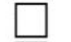 & Points of Contact w/ Clothing \\
\hline Thighs & E & (e.g waistband, undergarment) \\
\hline Lower legs & $\square$ & Groin \\
\hline Tops of Feet/Toes & & \\
\hline
\end{tabular}




\section{DERMATOLOGY LIFE OUALITY INDEX}

Hospita1 No:

Name:

Addres:
Date

Diagnosis:
DLQI

Score:



The aim of this questionnaire is to m easure how much your skin problem has affected your life OVER THE LAST WEEK. Please tick $\square$ one box for each question.

1. Over the last week, how itchy, sore, painful or stinging has your skin been?

2. Over the last week, how embarrassed or self conscious have you been because of your skin?

3. Over the last week, how much has your skin interfered with you going shopping or looking after your h ome or garden?

4. Over the last week, how much has your skin influenced the clothes you wear?

5. Over the last week, how much has your skin affected arty social or leisure activities?

6. Over the last week, how much has your skin made it difficult for you to do any sport?

7. Over the last week, has your skin prevented you from working or studying?

If "No", over the last week how much has your skin been a problem at work or studying?

8. Over the last week, how much has your skin created problems with your partner or any of your close friends or relatives?

9. Over the last week, how much has your skin caused any sexual difficulties?

10. Over the last week, how much of a problem has the treatment for your skin ben, for example by making your home messy, or by taking up time?
Very much

A bt

A little

Not at all

Very much

A bt

A little

Not at all

Very much

A bt

A little

Not at all

Not relevant

Very much

A bt

A little

Not at all

Not relevant

Very much

A bt

A little

Not at all

Not relevant

Very much

A bt

A little

Not at all

Not relevant

Yes

No

Not relevant

A bt

A little

Not at all

Very much

A bt

A little

Not at all

Not relevant $\square$

Very mudh

A bt

A little

Not at all

Not relevant

Very much

A bt

A little

Not at all

Please check you h ave answered EVERY question. Thank you.

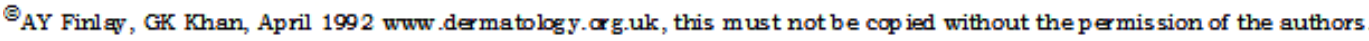

Note: The prior consent of the copyright holder was taken. 